Radiologe 2021 - 61:979-985

https://doi.org/10.1007/s00117-021-00921-4

Angenommen: 15. September 2021

Online publiziert: 18. Oktober 2021

(c) Springer Medizin Verlag $\mathrm{GmbH}$, ein Teil von

Springer Nature 2021

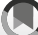

\section{Strukturierte Befundung in der Radiologie}

\section{Sicht der deutschen und europäischen Fachgesellschaften}

\author{
Elmar Kotter ${ }^{1} \cdot$ Daniel Pinto dos Santos ${ }^{2}$ \\ ' Klinik für Diagnostische und Interventionelle Radiologie, Universitätsklinikum Freiburg, Freiburg, \\ Deutschland \\ ${ }^{2}$ Institut für Diagnostische und Interventionelle Radiologie, Uniklinik Köln, Köln, Deutschland
}

\section{Zusammenfassung}

\section{In diesem Beitrag}

\section{- Aktivitäten der Fachgesellschaften Aktivitäten der Deutschen Röntgengesell- schaft • European Society of Radiology • Subspecialty Societies der ESR \\ - Industrielle Aktivitäten}

Zahlreiche Publikationen belegen den herausragenden Wert einer strukturierten Befundung sowohl für die Kommunikation mit zuweisenden klinischen Kollegen als auch für die Weiterverwendung der Befunddaten in anderen Kontexten. Obwohl das Thema bereits seit vielen Jahren in der Radiologie bekannt ist, hat sich die strukturierte Befundung noch nicht flächendeckend in der klinischen Routine etablieren können. Alle größeren radiologischen Fachgesellschaften haben sich klar für die strukturierte Befundung ausgesprochen und verfolgen etliche Initiativen auf diesem Gebiet. Dazu zählt der Aufbau frei zugänglicher Sammlungen von Befundvorlagen und die Qualitätssicherung derselben sowie die Pflege und Entwicklung standardisierter Begriffslexika. Im vorliegenden Artikel werden insbesondere die Aktivitäten der Deutschen Röntgengesellschaft und der European Society of Radiology dargestellt sowie ein kurzer Überblick über Vor- und Nachteile und verfügbare Ressourcen gegeben.

\section{Schlüsselwörter}

Befundvorlagen · Befundungssysteme · Kommunikation · Qualitätssicherung · Künstliche Intelligenz

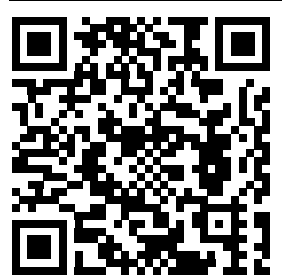

QR-Code scannen \& Beitrag online lesen
Auch wenn zahlreiche Publikationen der letzten Jahre den herausragenden Wert einer strukturierten Befundung nahelegen stellt auch heute noch der unstrukturierte radiologische Befundbericht, der in freier und prosaähnlicher Form verfasst wird, den klinischen Standard dar [4-6, 13, 21-24].

Interessanterweise ist das Thema aber in der Radiologie bereits seit langer Zeit präsent. So wurden bereits in den 1920er Jahren erste Stimmen laut, die eine standardisierte und strukturiertere Form der Befunderstellung als den üblichen Freitext-Befund forderten [14]. Anlass war damals wie heute die bisweilen fehlende Vergleichbarkeit von Befunden unterschiedlicher Radiologen sowie die Tatsache, dass sich radiologische Befundberichte nicht zu weitergehender wissenschaftlicher Auswertung eignen. Insbesondere in den letzten 15 Jahren positionierte sich eine Vielzahl radiologischer
Fachgesellschaften (u. a. American College of Radiology [ACR], Radiological Society of North America [RSNA], European Society of Radiology [ESR] und Deutsche Röntgengesellschaft [DRG]) noch einmal klar und sprachen sich für eine flächendeckende Einführung und Nutzung strukturierter Befundvorlagen aus [11, 12, 19, 23].

Trotz der in der Literatur umfassend dargestellten Vorteile (•Tab. 1) und der Unterstützung durch die Fachgesellschaften konnte sich die strukturierte Befundung noch nicht in der täglichen radiologischen Praxis durchsetzen. Einer der Hauptgründe hierfür liegt in der im Vergleich zum konventionellen Diktat aufwändigeren Erfassung des Befundberichts mittels strukturierter Vorlagen. Praktisch alle auf dem Markt verfügbaren Systeme zur strukturierten Befunderfassung arbeiten bislang mit Formularen, welche mit Maus und Tastatur befüllt werden müssen. Damit kann 


\begin{tabular}{|c|c|}
\hline Vollständigkeit & $\begin{array}{l}\text { Durch die vorgegebenen Elemente eines strukturierten Befundes wird die } \\
\text { Vollständigkeit der Befunde sichergestellt und damit implizite Informations- } \\
\text { übermittlung vermieden }\end{array}$ \\
\hline $\begin{array}{l}\text { Standardisierter } \\
\text { Befundinhalt }\end{array}$ & $\begin{array}{l}\text { Im Idealfall ist die Befundvorlage im Vorfeld mit den Kollegen der Fachklini- } \\
\text { ken abgestimmt (konsentiert) und sollte deren Bedürfnissen für die Steue- } \\
\text { rung der Behandlung entsprechen }\end{array}$ \\
\hline $\begin{array}{l}\text { Leitfaden für } \\
\text { die Befundung }\end{array}$ & $\begin{array}{l}\text { Angehende, in einem Gebiet noch nicht erfahrene Kollegen, werden durch } \\
\text { die Befundvorlagen unterstützt. Die Vorlage stellt die relevanten Fragen/ } \\
\text { Befundinhalte übersichtlich dar und dient so als Leitfaden }\end{array}$ \\
\hline Kommunikation & $\begin{array}{l}\text { Strukturiert erfasste Befundinhalte können je nach Empfänger in unter- } \\
\text { schiedlich dargestellt werden (bspw. Fließtext, tabellarische Darstellung, } \\
\text { für Patienten optimierte Sprache, etc.) }\end{array}$ \\
\hline $\begin{array}{l}\text { Semantische } \\
\text { Verbindung } \\
\text { zwischen Be- } \\
\text { fund und Bild }\end{array}$ & $\begin{array}{l}\text { Die Verwendung der strukturierten Befundung erleichtert die Erstellung von } \\
\text { Hyperlinks zwischen Befund und Bildmaterial. Schließlich erlaubt die Ver- } \\
\text { wendung standardisierter Lexika oder Ontologien zukünftig die semantische } \\
\text { Erschließung des Inhalts radiologischer Befunde }\end{array}$ \\
\hline $\begin{array}{l}\text { Zeitlicher Ver- } \\
\text { lauf }\end{array}$ & $\begin{array}{l}\text { Die Verwendung der strukturierten Befundung erleichtert die Darstellung } \\
\text { von Verläufen, z. B. für onkologische Patienten, in einer Zeitschiene erheblich }\end{array}$ \\
\hline $\begin{array}{l}\text { Actionable } \\
\text { Reports }\end{array}$ & $\begin{array}{l}\text { Die Verwendung der strukturierten Befundung erlaubt die automatische Aus- } \\
\text { lösung von Ereignissen (z. B. Setzen eines Flags zur Verfolgung eines patho- } \\
\text { logischen Befunds) und damit eine bessere Nachverfolgung von Befunden }\end{array}$ \\
\hline $\begin{array}{l}\text { Sekundäre Da- } \\
\text { tennutzung/ } \\
\text { Machine Learn- } \\
\text { ing }\end{array}$ & $\begin{array}{l}\text { Mittels strukturierter Befundung erhobene und in einer Datenbank gespei- } \\
\text { cherte Befunde können für wissenschaftliche Auswertungen genutzt werden. } \\
\text { Insbesondere können die erhobenen Befunde auch als (zumindest schwache) } \\
\text { Annotation für Machine Learning genutzt werden }\end{array}$ \\
\hline $\begin{array}{l}\text { Korrelation } \\
\text { mit anderen } \\
\text { Datenquellen }\end{array}$ & $\begin{array}{l}\text { Die Verwendung der strukturierten Befundung in verschiedenen Disziplinen } \\
\text { erlaubt die Korrelation der erhobenen Befunde. So ist z. B. eine Feedback- } \\
\text { Schleife von der Pathologie an die Radiologie denkbar }\end{array}$ \\
\hline
\end{tabular}

die Erfassung des strukturierten Befundes nicht erfolgen, während der Radiologe die Untersuchung auswertet und mit den Bilddaten interagiert. Hinzu kommt, dass der Mehrwert einer strukturierten Befundung vor allem bei der Befundung von Folgeuntersuchungen offensichtlich wird, der initial befundende Radiologe inn also nicht unmittelbar wahrnimmt. Bei Initialbefunden wird der Mehrwert strukturierter Befundberichte v. a. in der besseren Kommunikation mit Zuweisern offenbar [7, 26, 28, 29].

\section{Aktivitäten der Fachgesellschaften}

\section{Aktivitäten der Deutschen Röntgengesellschaft}

Die Deutsche Röntgengesellschaft (DRG) definierte im Jahr 2017 die strukturierte Befundung als eines der Leuchtturmprojekte für die kommenden Jahre. Dabei gab sie sich insbesondere den Auftrag, in den folgenden Jahren eine Sammlung qualitätsgesicherter strukturierter Befundvorlagen zu schaffen, die unter freier Lizenz in einem interoperablen Format zur Verfügung gestellt werden sollten [2]. In Anlehnung an die bereits bestehenden Initiativen von ESR und RSNA wurde hierzu IHE MRRT (Integrating the Healthcare Enterprise - Management of Radiology Report Templates) gewählt $[15,17]$. Parallel hierzu verpflichtete sich die DRG, eine deutschsprachige Übersetzung des RadLex der RSNA zur Verfügung zu stellen, um so auch für die in den Befundvorlagen definierten Inhalte eine möglichst einheitliche und international vergleichbare Terminologieverwenden zu können.

\section{Arbeitskreis Strukturierte Befundung}

Als im Jahr 2017 die operative Koordinierung der Initiative dem Arbeitskreis Strukturierte Befundung innerhalb der Arbeitsgemeinschaft Informationstechnologie (AGIT) der DRG übertragen wurde, lagen auf der englischsprachigen Seite radreport.org der RSNA über 200 Befundvorlagen vor, die bereits mehrere Millionen Male abgerufen worden waren (- Abb. 1). Im Gegensatz zu der Initiative der RSNA wurde aber beschlossen, dass die Erarbeitung von Befundvorlagen im Rahmen eines interdisziplinären Konsensprozesses erfolgen sollte.

Hierbei werden die medizinischen Inhalte zunächst in einer ersten Version durch die anderen jeweils organ- bzw. pathologiespezifischen Arbeitsgemeinschaften der DRG erarbeitet, wobei bereits relevante Leitlinien oder internationale Konsensempfehlungen berücksichtigt werden sollen. Diese vorläufigen Befundvorlagen werden dann von Vertretern der DRG mit anderen klinischen Fachgesellschaften diskutiert, um eine möglichst gute Ausrichtung an den Bedürfnissen der zuweisenden Kollegen zu gewährleisten [16]. Erst nach Abschluss dieses Prozesses werden dann die so einvernehmlich beschlossenen Befundvorlagen öffentlich auf einer eigens eingerichteten Webseite (http://www.befundung.drg.de) zur Verfügung gestellt (• Abb. 2). Die Befundvorlagen werden unter einer Creative Commons Lizenz (CC-BY-4.0 - d. h. Download, Bearbeitung und Weiterverteilung der Befundvorlagen sind unter Nennung der DRG als Urheber erlaubt) allen Interessierten kostenlos zur Verfügung gestellt. Auch eine kommerzielle Nutzung ist unter dieser Lizenz explizit gestattet. Gemeinsam mit der Verwendung eines herstellerunabhängigen, interoperablen Formats, soll Herstellern von Befundungssystemen so eine niederschwellige Möglichkeit geboten werden, die Befundvorlagen der DRG zu verwenden und in ihre Systeme zu integrieren. Alle veröffentlichten Befundvorlagen sollen im weiteren Verlauf einer kontinuierlichen Überprüfung und - falls nötig - Aktualisierung unterzogen werden. Die Entwicklung und Aktualisierung der Befundvorlagen wird dabei über ein offen zugängliches Versionsverwaltungssystem (https://github.com/DRGagit/ak_ befundung) organisiert. Darüber hinaus besteht die Möglichkeit, Rückmeldungen per E-Mail an eine zentrale Adresse (strukturierte-befundung@drg.de) zu geben.

Zum Zeitpunkt der Redaktion liegen 24 konsensbasierte und qualitätsgesicherte Befundvorlagen zu einer Vielzahl von Pathologien vor. Ein besonderer Schwerpunkt der veröffentlichten Befundvorlagen liegt dabei auf onkologischen Fragestellungen (bspw. zum Staging bei Rektumkarzinom und Pankreasneoplasien [20]) sowie bildgebenden Verfahren 

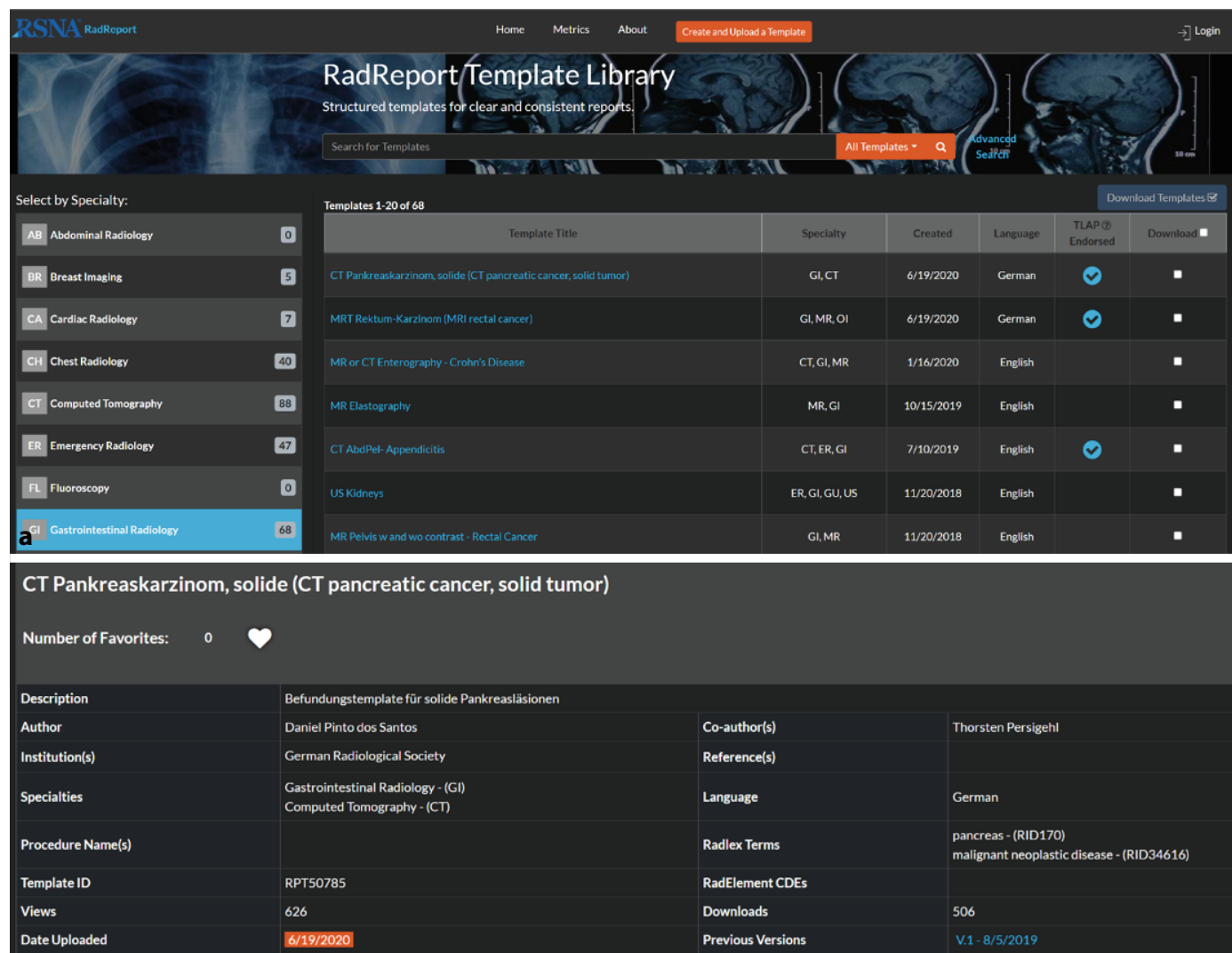

Template Displayed Below

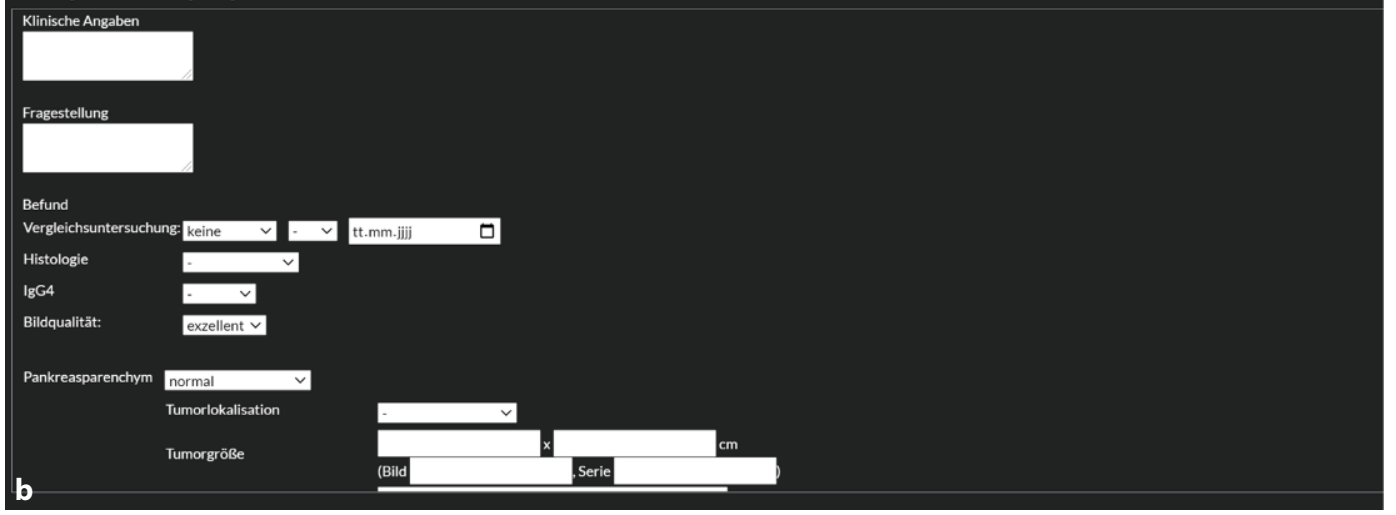

Abb. $1 \varangle$ a RadReport.org. b Deutschsprachiges Report Template in RadReport.org. (Mit freundlicher Genehmigung, @ Radiological Society of North America. Alle Rechte vorbehalten)

in der Kardiodiagnostik [8]. Diese wurden im Rahmen mehrtägiger Arbeitstreffen mit Vertretern der Deutschen Gesellschaft für Allgemein- und Viszeralchirurgie, der Deutschen Krebsgesellschaft bzw. Vertreter aus Herzchirurgie und Kardiologie konsentiert. Unter anderem bedingt durch die der Coronavirus-Pandemie geschuldeten Einschränkungen, konnten zuletzt keine weiteren Konsensusmeetings stattfinden, einzelne Befundvorlagen konnten aber trotzdem in entsprechenden Onlinekonferenzen erarbeitet werden. Für die kommenden Jahre ist eine Reihe weiterer Arbeitstreffen zu onkologischen Fragestel- lungen geplant, die Finanzierung erfolgt hierbei u.a. aus Mitteln der Deutschen Krebsgesellschaft.

Bewusst wurde seitens des Arbeitskreises Strukturierte Befundung die Entscheidung getroffen, keine umfassenden Befundvorlagen für einzelne Untersuchungen oder Modalitäten zu erarbeiten, mithilfe derer sich verschiedene klinische Fragestellungen beantworten ließen. Stattdessen soll möglichst je klinischer Fragestellung eine dedizierte Befundvorlage definiert werden, die als Minimalstandard verstanden werden könnte, und erlauben sollte, einen qualitativ hochwertigen und klinisch relevanten Befundbericht zu erstellen $[1,18,27]$. Auch soll nicht das Ziel sein, für jede mögliche Fragestellung und jedes denkbare klinische Szenario eine entsprechende Befundvorlage anzubieten. Vielmehr soll der Fokus der Entwicklung auf solchen Vorlagen liegen, die entweder durch die Häufigkeit der entsprechenden Fragestellung herausstechen (wie bspw. Magnetresonanztomographie [MRT] der Lendenwirbelsäule [LWS] bei V.a. Bandscheibenvorfall oder kraniale Computertomographie [cCT] nativ bei V.a. ischämischen Schlaganfall) oder bei denen in besonderem Maße die Qualität 

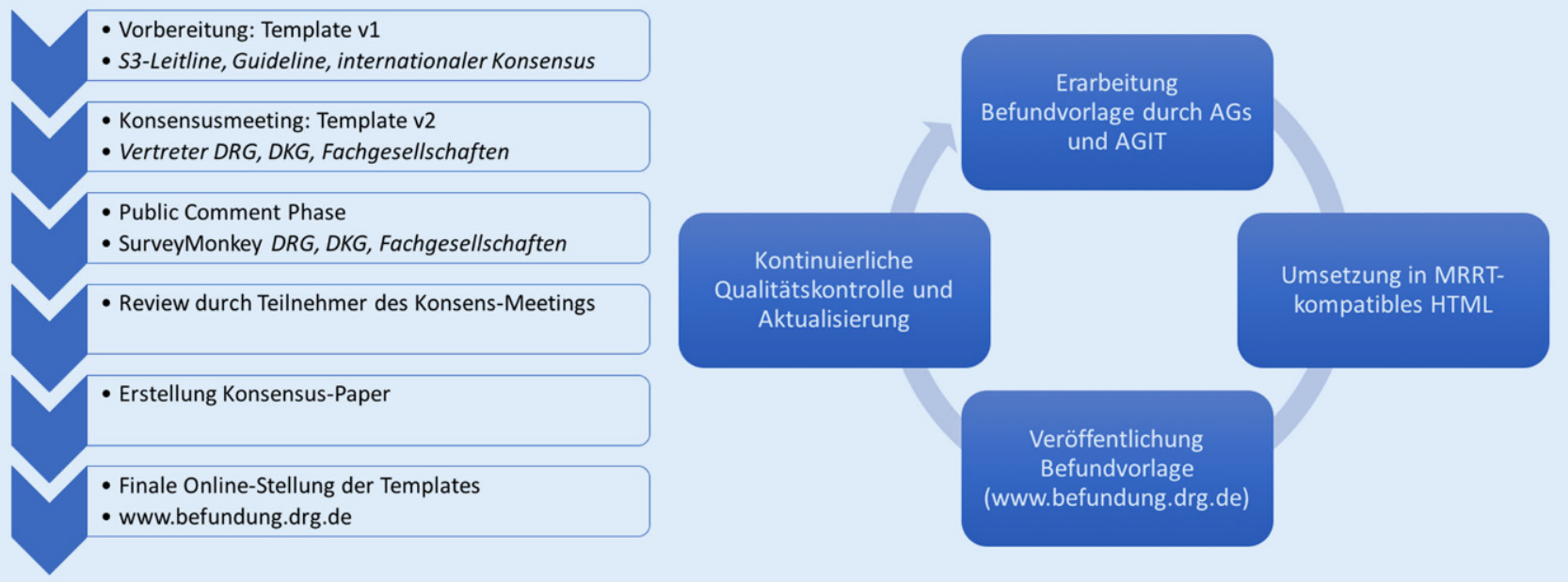

Abb. $2 \Delta$ Prozess der Erstellung qualitätsgesicherter und konsensbasierter Befundvorlagen sowie anschließende kontinuierliche Qualitätskontrolle und Aktualisierung. (Aus [23])

der entstehenden Befundberichte gesteigert werden kann (wie bspw. seltene aber komplexe Fragestellungen wie MRT des Herzens bei Siderose oder Primärstaging bei V.a. Pankreaskarzinom).

Der Arbeitskreis Strukturierte Befundung führt mit einzelnen Herstellern bereits Gespräche, um die Akzeptanz der strukturierten Befunderstellung und deren Integration in bestehende Infrastrukturen und klinische Abläufe zu fördern. Da nur wenige kommerziell verfügbare Lösungen bisher die unmittelbare Nutzung MRRT-konformer Befundvorlagen erlauben, werden vermutlich zunächst noch individuelle Umsetzungen der Befundvorlagen in proprietäre Formate gefunden werden. Für Interessierte, die trotzdem gerne direkt die Nutzung der DRG-Befundvorlagen ausprobieren wollen, hat die AG Informationstechnologie (AGIT) ein kleines webbasiertes Tool (EasyRad, IFTM $\mathrm{GmbH}$, Solingen) zur Verfügung gestellt. Diese Applikation erlaubt zumindest eine limitierte Interaktion mit der Befundvorlage, und die so erstellten Befundberichte können über die Zwischenablage in das jeweilige Radiologieinformationssystem übertragen werden.

\section{Übersetzung RadLex}

Wie bereits oben erwähnt, wurde parallel zum Arbeitskreis Strukturierte Befundung eine Initiative zur Übersetzung des RadLex ins Leben gerufen. Auch hier oblag die operative Koordination der AGIT. Diejewei- ligen organ- bzw. pathologiespezifischen Arbeitsgemeinschaften der DRG wurden im Übersetzungsprozess von Begriffen aus ihrem Themengebiet eingebunden und um Rückmeldungen und Korrekturen gebeten. Nach entsprechenden Qualitätssicherungsschritten konnte schließlich eine vollständige Übersetzung des englischen RadLex an die RSNA übergeben werden, sodass nun sowohl die englisch- als auch die deutschsprachigen Begriffe in der Datenbank verfügbar sind. Auch diese Begriffe können unter einer entsprechenden Lizenz kostenfrei sowohl für kommerzielle als auch nichtkommerzielle Nutzung verwandt werden.

Bislang sind die RadLex-Termini nur in einzelne der Befundvorlagen der DRG integriert worden, dies soll aber zeitnah durch den Arbeitskreis Strukturierte Befundung ergänzt werden. Eine solche Verknüpfung von Befundinhalten zu eindeutigen RadLex-Codes könnte eine Vielzahl interessanter Möglichkeiten eröffnen, von automatisierten Übersetzungen der Befunde über institutionsübergreifende Auswertungen bis hin zu Anwendungen der künstlichen Intelligenz (KI). Aus Sicht der Deutschen Röntgengesellschaft stellt die strukturierte Befundung einen zentralen Baustein einer datengetriebenen Radiologie der Zukunft dar.

Eine Übersicht von Online-Ressourcen zeigt $\bullet$ Tab. 2 .

\section{European Society of Radiology}

Innerhalb der ESR verantwortet das eHealth and Informatics Subcommittee den gesamten Bereich "Imaging Informatics". Gemeinsam haben die ESR und das eHealth and Informatics Subcommittee die strukturierte Befundung schon vor über 10 Jahren als eines der Kernthemen der radiologischen IT definiert.

Das Thema der strukturierten Befundung wird innerhalb der ESR auf verschiedenen Ebenen behandelt: Die RSNA/ESR Structured Reporting Initiative wurde während des ECR 2013 gegründet mit dem Ziel, mehrsprachige Befundvorlagen zu erstellen. Diese Initiative hat sich zu einem Diskussionsforum unter Teilnahme aller nationalen Röntgengesellschaften sowie der Subspecialty Societies entwickelt. Das Template Library Advisory Panel (TLAP) ist ein gemeinsames Committee der ESR und der RSNA. TLAP verwaltet und steuert die Entwicklung von Befundvorlagen weltweit.

Ein zentraler Baustein ist hierbei die von der RSNA betriebene Plattform radreport.org (- Abb. 1), die als multilinguales Report Template Repository dient. Auf radreport.org kann jeder Nutzer Befundvorlagen einreichen, das TLAP überprüft dann in einem strukturierten Reviewprozess, ob die eingestellten Befundvorlagen den nötigen Mindestanforderungen genügen. Wenn dies der Fall ist, erhalten die Befundvorlagen den Status "TLAP endor- 


\begin{tabular}{|l|l|l|}
\hline \multicolumn{2}{|c|}{ Tab. 2 Online-Ressourcen zur strukturierten Befundung } \\
\hline $\begin{array}{l}\text { Befundvorlagen } \\
\text { der DRG }\end{array}$ & $\begin{array}{l}\text { https://www.befundung.drg.de/ } \\
\text { de-DE/3199/befundvorlagen/ }\end{array}$ & $\begin{array}{l}\text { Von der DRG zertifizierte Befundvorlagen. Betreut durch die Arbeitsgemeinschaft Informa- } \\
\text { tionstechnologie }\end{array}$ \\
\hline $\begin{array}{l}\text { Befundvorlagen } \\
\text { von RSNA und ESR }\end{array}$ & http://RadReport.org & $\begin{array}{l}\text { Von RSNA initiierte, inzwischen von dem gemeinsamen Template Library Advisory Panel } \\
\text { betreute Sammlung von Befundvorlagen }\end{array}$ \\
\hline $\begin{array}{l}\text { Common Data } \\
\text { Elements }\end{array}$ & https://www.radelement.org/ & $\begin{array}{l}\text { CDE sind Gruppen von standardisierten Fragen mit einem vorgegebenen Bereich von Ant- } \\
\text { wortmöglichkeiten. CDEs basieren auf RadLex und bilden wiederum Bausteine in Befund- } \\
\text { vorlagen }\end{array}$ \\
\hline RadLex & http://radlex.org/ & $\begin{array}{l}\text { RadLex ist eine radiologische Ontologie, in der über 45.000 radiologische Begriffe und } \\
\text { ihre Beziehungen untereinander definiert sind. RadLex wurde von der DRG vollständig ins } \\
\text { Deutsche übersetzt. Weitere Übersetzungen (z. B. Portugiesisch) sind in Vorbereitung }\end{array}$ \\
\hline $\begin{array}{l}\text { RadLex Bioportal } \\
\text { Browser }\end{array}$ & $\begin{array}{l}\text { http://bioportal.bioontology.org/ } \\
\text { ontologies/RADLEX/?p=classes\& } \\
\text { conceptid }\end{array}$ & $\begin{array}{l}\text { Darstellung von RadLex, Ontologie inklusive der Beziehungen zwischen den Begriffen und } \\
\text { Quervernetzung zu anderen Ontologien }\end{array}$ \\
\hline
\end{tabular}

\begin{tabular}{|l|l|}
\hline \multicolumn{2}{|l|}{ Tab. 3 Beispiele für Empfehlungen der Fachgesellschaften zur strukturierten Befundung } \\
\hline Subspecialty Society & Empfehlung \\
\hline European Society of Thoracic Imaging (ESTI) & $\begin{array}{l}\text { Befundvorlage für Lung Cancer Scree- } \\
\text { ning: https://www.myesti.org/content- } \\
\text { esti/uploads/ESTI-LCS-structured-report- } \\
\text { basic-template_2019-07-09.pdf }\end{array}$ \\
\hline $\begin{array}{l}\text { European Society of Gastrointestinal and Abdo- } \\
\text { minal Radiology (ESGAR) }\end{array}$ & $\begin{array}{l}\text { Empfehlung für die Befundung und Befund- } \\
\text { vorlage MRI Rektumkarzinom [3] }\end{array}$ \\
\hline European Society of Breast Imaging & BI-RADS [10] \\
\hline ESUR & PI-RADS [25, 31] \\
\hline $\begin{array}{l}\text { Society of Cardiovascular Computed Tomography } \\
\text { (SCCT), the American College of Radiology (ACR) } \\
\text { and the North American Society for Cardiovascu- } \\
\text { lar Imaging (NASCI) }\end{array}$ & CAD-RADS [9] \\
\hline
\end{tabular}

sed“. Zum Zeitpunkt der Redaktion waren auf RadReport.org 428 Befundvorlagen verfügbar, darunter 164 mit dem Status "TLAP endorsed". Befundvorlagen stehen auf der Plattform in unterschiedlichen Sprachen zur Verfügung, so zum Zeitpunkt der Redaktion u. a. 263 in Englisch, 27 auf Niederländisch, 26 auf Italienisch, und 9 auf Deutsch, darunter 5 mit dem Status "TLAP endorsed".

Ein vielbeachteter Artikel der RSNA/ESR Structured Reporting Initiative erschien 2015 in der Fachzeitschrift Insights into Imaging und stellte die Vorteile der strukturierten Befundung für die Radiologie eindrücklich und teils auch provokativ dar [4]. Mitglieder des eEhealth and Informatics Subcommittee haben 2018 im Auftrag der ESR einen Artikel zur strukturierten Befundung in der Radiologie veröffentlicht [12], welcher die offizielle Position der ESR wiedergibt: „Die Entwicklung vom konventionellen Prosa-Befund hin zur strukturierten Befundung wird [von der ESR] unterstützt. Diese Entwicklung kann nur im Rahmen einer internationalen
Kooperation stattfinden. Diese ermöglicht die Entwicklung und Akzeptanz von strukturierten Befundvorlagen, welche dem Bedarf entsprechend in verschiedene Sprachen übersetzt werden können und an lokale Gegebenheiten angepasst werden können." In dem Artikel wird hervorgehoben, dass die Implementierung von strukturierten Befundvorlagen in die radiologische Routinearbeit nur unter Mitwirkung der Industrie geschehen kann. Aufgrund der Dynamik der Entwicklung der strukturierten Befundung ist aktuell eine Version 2.0 des Positionspapiers der ESR in Arbeit.

Potenziell ergibt sich aus der Nutzung von Befundvorlagen in Kombination miteinem standardisierten Vokabular (RadLex) die Möglichkeit, Befunde ohne Ambiguitäten vollautomatisch von einer Sprache in eine andere zu übersetzen [30]. Dies setzt voraus, dass das entsprechende Vokabular in der Quell- und Zielsprache zur Verfügung steht (RadLex steht in einer deutschen Übersetzung zur Verfügung, eine Übersetzung ins Portugiesische befindet sich in Arbeit), und dass das entsprechende Report Template ebenfalls in beiden Sprachen zur Verfügung steht. Die Plattform radreport.org kann aktuell zwar Befundvorlagen in verschiedenen Sprachen verwalten, die Zuordnung von Befundvorlagen gleichen Inhalts aber unterschiedlicher Sprache ist aktuell jedoch noch nicht möglich, wird aber auf Anregung der ESR zukünftig implementiert werden.

\section{Subspecialty Societies der ESR}

Die European Society of Imaging Informatics (EuSoMII) unterstützt die Verbreitung der strukturierten Befundung und der dazugehörigen Werkzeuge. Strukturierte Befundung ist immer wieder Teil der Jahrestreffen und auch der von EuSoMII organisierten Weiterbildungen in Form von Webinaren. Wie auch in Bezug auf andere Themen steht die EuSoMII in stetigem Austausch sowohl mit der ESR als auch der USamerikanischen Schwestergesellschaft der EuSoMII, der Society of Medical Imaging Informatics (SIIM).

Strukturierte Befundung wird von nahezu allen affiliierten Subspecialty Societies der ESR unterstützt. Entsprechende Befundvorlagen wurden von diesen Gesellschaften sowohl in wissenschaftlichen Publikationen als auch als Empfehlungen auf ihren Websites veröffentlicht (• Tab. 3).

\section{Industrielle Aktivitäten}

Alle genannten Gesellschaften sind sich darin einig, dass die Umsetzung der strukturierten Befundung im radiologischen Alltag nur funktionieren kann, wenn die industriellen Partner Werkzeuge entwickeln, 
welche die Erstellung strukturierter Befunde mit einem minimalen Mehraufwand für den befundenden Radiologen ermöglichen. Auf dem deutschen Markt gibt es verschiedene Hersteller, die strukturierte Befundung verwenden oder deren Geschäftszweck die Nutzung und Verbreitung von Befundvorlagen ist. Mint Medical ist weit verbreitet für die standardisierte Befundung von Studienuntersuchungen v.a. im onkologischen Kontext. Hierbei werden typischerweise studienrelevante Klassifikationen wie RECIST, Cheson oder RANO genutzt. Für Studienuntersuchungen hat sich die strukturierte Befundung trotz des erhöhten Aufwands weitgehend durchgesetzt, da der erhöhte Aufwand i.d. R. vergütet wird. Auch ist hier hilfreich, dass der Aufwand v. a. bei der Erstbefundung eines Patienten erhöht ist, sich jedoch bei den häufigen Follow-up-Untersuchungen wieder deutlich reduziert. SmartReporting und RadioReport verfolgen den Ansatz, Systeme zur strukturierten Befundung auch außerhalb von klinischen Studien verfügbar zu machen, wobei bislang keines der Systeme die nahtlose Nutzung der von den wissenschaftlichen Gesellschaften getragenen Standards, also die Nutzung der Befundvorlagen in den Repositories der DRG oder der ESR/RSNA, ermöglicht. Alle auf dem deutschen Markt verfügbaren Systeme erstellen den strukturierten Befund über eine grafische Benutzeroberfläche, welche mit der Maus zu bedienen ist, was den Radiologen zwangsläufig von der Betrachtung des Bildmaterials ablenkt. Neben den genannten, auf strukturierte Befundung spezialisierte Firmen befassen sich auch die meisten RISund PACS-Hersteller mit der Thematik.

Ähnlich wie die Entwicklung von Gaming-Grafikkarten das Deep Learning überhaupt erst ermöglicht hat, kann erwartet werden, dass verbesserte Spracherkennungstechnologien aus dem Consumerbereich auch in der Medizin Einzug halten werden. Diese könnten dann ein Ausfüllen der Befundvorlagen durch das Diktat des Radiologen möglich machen.

\section{Fazit für die Praxis}

- Die strukturierte Befundung weist, inzwischen durch zahlreiche Publikationen belegte, Vorteile gegenüber der konventionellen radiologischen Freitext-Befundung auf und wird von allen relevanten radiologischen Fachgesellschaften unterstützt.

- Der Einsatz strukturierter Befundung in der klinischen Routine wird heute noch durch den erhöhten Aufwand bei der Befundung behindert, könnte aber in $\mathrm{Zu}$ kunft durch gezielte Anregungen (z.B. eine erhöhte Vergütung) gefördert werden.

- Auch der Einsatz innovativer Technologien zur Vereinfachung der Erstellung von strukturierten Befunden könnten helfen, den Mehraufwand zu verringern.

\section{Korrespondenzadresse}

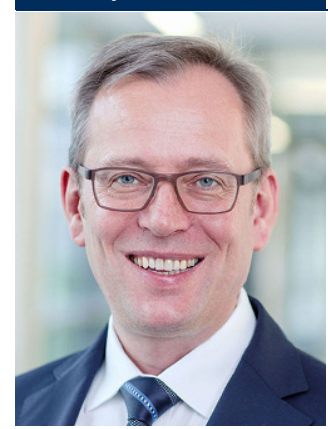

Prof. Dr. Elmar Kotter

Klinik für Diagnostische und Interventionelle Radiologie, Universitätsklinikum Freiburg Hugstetter Str. 55, 79106 Freiburg, Deutschland elmar.kotter@uniklinik-freiburg.de

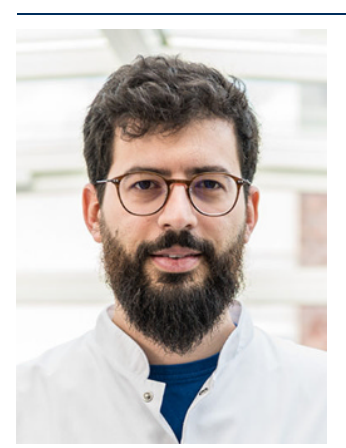

PD Dr. Daniel Pinto dos Santos

Institut für Diagnostische und Interventionelle Radiologie, Uniklinik Köln

Kerpener Str. 62, 50937 Köln, Deutschland daniel.pinto-dos-santos@uk-koeln.de

\section{Einhaltung ethischer Richtlinien}

Interessenkonflikt. E. Kotter und D. Pinto dos Santos geben an, dass kein Interessenkonflikt besteht.

Für diesen Beitrag wurden von den Autoren keine Studien an Menschen oder Tieren durchgeführt. Für die aufgeführten Studien gelten die jeweils dort angegebenen ethischen Richtlinien.

\section{Literatur}

1. Anderson TJT, Lu N, Brook OR (2017) Diseasespecific report templates for your practice. J Am Coll Radiol 14:1055-1057. https://doi.org/10. 1016/j.jacr.2016.12.019

2. Bauer J, Rohner-Rojas S, Holderried M (2020) Einrichtungsübergreifende Interoperabilität: Herausforderungen und Grundlagen für die technische Umsetzung. Radiologe 60:334-341. https://doi. org/10.1007/s00117-019-00626-9

3. Beets-Tan RGH, Lambregts DMJ, Maas $M$ et al (2018) Magnetic resonance imaging for clinical management of rectal cancer: updated recommendations from the 2016 European society of gastrointestinal and abdominal radiology (ESGAR) consensus meeting. Eur Radiol 28:1465-1475. https://doi.org/10.1007/s00330-017-5026-2

4. Bosmans JML, Neri E, Ratib O, Kahn CE (2015) Structured reporting: a fusion reactor hungry for fuel. Insights Imaging 6:129-132. https://doi.org/ 10.1007/s13244-014-0368-7

5. Bosmans JML, Peremans L, Menni M et al (2012) Structured reporting: if, why, when, how - and at what expense? Results of a focus group meeting of radiology professionals from eight countries. Insights Imaging 3:295-302. https://doi.org/10. 1007/s13244-012-0148-1

6. Bosmans JML, Weyler JJ, De Schepper AM Parizel PM (2011) The radiology report as seen by radiologists and referring clinicians: results of the COVER and ROVER surveys. Radiology 259:184-195. https://doi.org/10.1148/radiol. 10101045

7. Brook OR, Brook A, Vollmer CM et al (2015) Structured reporting of multiphasic CT for pancreatic cancer: potential effect on staging and surgical planning. Radiology 274:464-472. https:// doi.org/10.1148/radiol.14140206

8. Bunck AC, Baeßler B, Ritter C et al (2019) Structured reporting in cross-sectional imaging of the heart: reporting templates for CMR imaging of cardiomyopathies (myocarditis, dilated cardiomyopathy, hypertrophic cardiomyopathy, arrhythmogenic right ventricular cardiomyopathy and siderosis). Rofo. https://doi.org/10.1055/a0998-4116

9. Cury RC, Abbara S, Achenbach S et al (2016) CADRADSTM coronary artery disease-reporting and data system. An expert consensus document of the society of cardiovascular computed tomography (SCCT), the American college of radiology (ACR) and the north American society for cardiovascular imaging (NASCl). Endorsed by the American college of cardiology.J Cardiovasc Comput Tomogr 10:269-281. https://doi.org/10.1016/j.jcct.2016. 04.005

10. D'Orsi C, Sickles EA, Mendelson E, Morris E (2013) ACR BI-RADS ${ }^{\circledR}$ Atlas, breast imaging reporting and data system

11. Dunnick NR, Langlotz CP (2008) The radiology report of the future: a summary of the 2007 intersociety conference. J Am Coll Radiol 5:626-629. https://doi.org/10.1016/j.jacr.2007.12.015

12. European Society of Radiology (2018) ESR paper on structured reporting in radiology. Insights Imaging 9:1-7. https://doi.org/10.1007/s13244017-0588-8

13. Hackländer T (2013) Strukturierte Befundung in der Radiologie. Radiologe 53:613-617. https://doi. org/10.1007/s00117-013-2493-6

14. Hickey P (1922) Standardization of Roentgen-ray reports. AJR Am J Roentgenol 9:422-425 
15. IHE Radiology Technical Committee (2018) IHE radiology technical framework supplement-management of radiology report templates (MRRT)

16. Kahn CE, Heilbrun ME, Applegate KE (2013) From guidelines to practice: how reporting templates promote the use of radiology practice guidelines. J Am Coll Radiol 10:268-273. https://doi.org/10. 1016/j.jacr.2012.09.025

17. Langlotz CP (2012) IHE radiology (RAD) white paper-management of radiology report templates (MRRT)

18. Mamlouk MD, Chang PC, Saket RR (2018) Contextual radiology reporting: a new approach to neuroradiology structured templates. AJNR Am J Neuroradiol. https://doi.org/10.3174/ajnr.A5697

19. Morgan TA, Heilbrun ME, Kahn CE (2014) Reporting initiative of the radiological society of north america: progress and new directions. Radiology 273:642-645. https://doi.org/10.1148/radiol. 14141227

20. Persigehl T, Baumhauer M, Baeßler B et al (2020) Structured reporting of solid and cystic pancreatic lesions in CT and MRI: consensus-based structured report templates of the German society of radiology (DRG). Rofo 192:641-656. https://doi. org/10.1055/a-1150-8217

21. Pinto Dos Santos D, Baeßler B (2018) Big data, artificial intelligence, and structured reporting. Eur Radiol Exp 2:42. https://doi.org/10.1186/s41747018-0071-4

22. Pinto Dos Santos D, BrodehI S, Baeßler B etal (2019) Structured report data can be used to develop deep learning algorithms: a proof of concept in ankle radiographs. Insights Imaging 10:93. https:// doi.org/10.1186/s13244-019-0777-8

23. Pinto Dos Santos D, Hempel J-M, Mildenberger $P$ et al (2019) Structured reporting in clinical routine. Rofo 191:33-39. https://doi.org/10.1055/a-06363851

24. Pinto Dos Santos D, ScheiblS, Arnhold Getal (2018) A proof of concept for epidemiological research using structured reporting with pulmonary embolism as a use case. Br JRadiol. https://doi.org/ 10.1259/bjr.20170564

25. Röthke $M$, Blondin $D$, Schlemmer H-P, Franiel $T$ (2013) PI-RADS-Klassifikation: Strukturiertes Befundungsschema für die MRT der Prostata. Rofo 185:253-261. https://doi.org/10.1055/s-00321330270

26. Sahni VA, Silveira PC, Sainani NI, Khorasani R (2015) Impact of a structured report template on the quality of MRI reports for rectal cancer staging. AJR Am JRoentgenol 205:584-588. https://doi.org/10. 2214/AJR.14.14053

27. Samartine S, White L, McKeon D, Becker M (2015) Enhancing structured reporting:improving quality by tailoring the report to the clinical scenario. J Am Coll Radiol 12:845-847. https://doi.org/10.1016/j. jacr.2015.05.026

28. Schoeppe F, Sommer WH, Nörenberg D et al (2018) Structured reporting adds clinical value in primary CT staging of diffuse large B-cell lymphoma. Eur Radiol 28:3702-3709. https://doi.org/10.1007/ s00330-018-5340-3

29. Schwartz LH, Panicek DM, Berk AR et al (2011) Improving communication of diagnostic radiology findings through structured reporting. Radiology 260:174-181. https://doi.org/10.1148/radiol. 11101913

30. Sobez LM, Kim SH, Angstwurm M et al (2019) Creating high-quality radiology reports in foreign languages through multilingual structured report-

\section{Structured reporting in radiology. German and European radiology societies' point of view}

Numerous publications have shown the value of structured reporting in communication with referring physicians and for further usage of clinical report data in other contexts. Despite the topic being present and known in radiology for many years now, widespread adoption of structured reporting in clinical routine is still lacking. All major radiological societies have published position statements in favor of structured reporting and are pursuing various related initiatives. Among those are the development and the maintenance of openly available structured report template repositories as well as quality control of report templates and development of standardized terminologies. This article highlights the efforts of the German Radiological Society and the European Society of Radiology on the topic of structured reporting and provides an overview of pros and cons as well as publicly available resources.

\section{Keywords}

Report templates $\cdot$ Reporting systems $\cdot$ Communication $\cdot$ Quality assurance $\cdot$ Artificial intelligence

ing. Eur Radiol. https://doi.org/10.1007/s00330019-06206-8

31. Turkbey B, Rosenkrantz AB, Haider MA et al (2019) Prostate imaging reporting and data system version 2.1: 2019 update of prostate imaging reporting and data system version 2 . Eur Urol 76:340-351. https://doi.org/10.1016/j.eururo. 2019.02.033 